\title{
Ixabepilone as monotherapy or in combination for the treatment of advanced breast cancer
}

\author{
This article was published in the following Dove Press journal: \\ Breast Cancer:Targets and Therapy \\ 22 May 2010 \\ Number of times this article has been viewed
}

\section{Erin M Bertino \\ Bhuvaneswari Ramaswamy \\ Department of Internal Medicine, Division of Hematology-Oncology, The Ohio State University Medical Center, Columbus, Ohio, USA}

\begin{abstract}
Advanced breast cancer represents a therapeutic challenge for oncologists. Chemotherapy with anthracyclines and taxanes has improved survival in this setting, but resistant and refractory disease is common. Ixabepilone, an epothilone, is a recently approved chemotherapeutic agent, both as a single agent and in combination with capecitabine, with efficacy in patients with resistant advanced disease. In this review, the distinctive properties of this drug are discussed, as well as the clinical evidence of efficacy. Ongoing clinical trials are exploring the role of ixabepilone in several clinical settings: neoadjuvant, adjuvant, and novel combinations.
\end{abstract}

Keywords: chemotherapy, solid tumor, metastatic disease, neoadjuvant chemotherapy

\section{Introduction}

Breast cancer incidence for 2009 is estimated at over 190,000 women with over 40,000 estimated deaths. ${ }^{1}$ With improving therapeutic options, patients are living longer, even with advanced disease. Despite these advances, however, median survival in this setting is approximately 2 to 3 years. ${ }^{2}$ Anthracyclines and taxanes remain effective and are the most commonly used agents in both adjuvant and metastatic settings. Although these agents are effective, a substantial number of tumors develop resistance to these agents, and newer agents that can circumvent pathways of resistance are needed to improve outcomes. In the setting of resistant metastatic disease, standard treatment produces diminishing responses.

Ixabepilone is an epothilone, a family of microtubule-targeted chemotherapeutic agents. Ixabepilone is currently approved for use in metastatic or locally advanced breast cancer resistant or refractory to taxane and anthracycline therapy. It is approved in combination with capecitabine or as a single agent. In this review, the development, pharmacology, clinical trials, toxicities, and future directions of ixabepilone will be discussed.

\section{Background Epothilones}

Epothilones are a family of macrolides derived from the myxobacterium Sorangium cellulosum. In early experiments, epothilones demonstrated an ability to induce microtubule polymerization and led to mitotic arrest and apoptosis in a manner similar to taxanes. ${ }^{3,4}$ Epothilone A and B were both tested in vitro and demonstrated 
taxane-like properties with superior cytotoxic potency against taxane-resistant cell lines. ${ }^{3}$ Naturally occurring epothilone B, however, demonstrated unfavorable pharmacokinetics and poor metabolic stability during testing on animal models in vivo. ${ }^{5,6}$ This finding led to the development of a semisynthetic epothilone B analog, ixabepilone.

\section{Relationship of ixabepilone to taxanes}

Ixabepilone is a microtubule-targeted agent with many similarities to taxanes but also with several clinically relevant differences. Like taxanes, ixabepilone acts to stabilize microtubules, disrupting the normal dynamic and arresting the cell cycle at the $\mathrm{G}_{2}-\mathrm{M}$ transition. Ixabepilone also has limited solubility in water and requires reconstitution with Cremophor EL ${ }^{\circledR}$ for infusion. ${ }^{7}$ Ixabepilone competitively binds at the same site as paclitaxel but with a potency approximately 2.5-fold greater than paclitaxel. Although this binding is competitive, epothilones interact biologically with the binding pocket in a unique manner which is different from the interaction of taxanes at the same site. ${ }^{8}$ Additional distinctive qualities of ixabepilone include ability to maintain cytotoxic activity despite the presence of multi-drug resistant proteins or tubulin mutations. $^{3,5,9,10}$

Specific mechanisms of taxane-resistance addressed by ixabepilone include overexpression of multi-drug resistant P-glycoprotein and tubulin mutations or overexpression. P-glycoprotein acts as a drug efflux protein, effectively pumping taxanes out of cells to impair cytotoxic activity. Tubulin-related taxane resistance in breast cancer has been linked to overexpression of $\beta$-III-tubulin. In preclinical evaluation, ixabepilone demonstrated activity against taxane-resistant cell lines expressing both of these mechanisms. The activity of ixabepilone against taxaneresistant malignancies is a cardinal feature. .,9-11 $^{5}$

\section{Early studies}

With encouraging pre-clinical evidence of ixabepilone activity, phase I testing was initiated to determine the maximum-tolerated dose (MTD) and pharmacokinetics. Several dosing schedules were tested: weekly, once every 21 days, and days 1 to 3 or 1 to 5 every 21 days. The primary dose-limiting toxicities (DLT) in all these studies were neuropathy, myelosuppression and fatigue (Figure 1).

Weekly dosing has been tested in several variations in phase I trials. Awada et al reported results of a phase I trial using 2 different weekly dosing schedules: a 30-minute intravenous (IV) infusion weekly for a 21-day cycle and a 60 -minute IV infusion days 1,8 , and 15 of a 28 -day cycle. ${ }^{12}$ Evaluation of safety, toxicity, and determination of MTD/ recommended phase II dose were the primary objectives of the trial. The study enrolled 86 patients with advanced, refractory solid tumors, including breast cancer; however, exact number of breast cancer patients was not reported. The MTD was $25 \mathrm{mg} / \mathrm{m}^{2}$ for the 21-day cycle and $20 \mathrm{mg} / \mathrm{m}^{2}$ for the 28-day cycle. The primary DLT were fatigue (grade 3 , $25 \%$ at MTD) and sensory peripheral neuropathy (grade 3 to $4,8 \%$ to $10 \%$ at MTD). Partial responses were observed in 5 patients $(5 \%) .^{12}$

Several studies have also evaluated a dosing schedule of once every 3 weeks. The first published results included 25 patients with advanced solid tumors, including 6 patients with breast cancer. All of the patients had received prior chemotherapy; 11 patients had received taxanes. Ixabepilone was administered as an IV infusion on day 1 of a 21-day cycle. The MTD was determined to be $50 \mathrm{mg} / \mathrm{m}^{2}$ and the recommended phase II dose was $40 \mathrm{mg} / \mathrm{m}^{2}$. Neutropenia $\geq$ grade 3 was the primary DLT. Additional toxicities included fatigue (all grades: 73\%), nausea (all grades: 52\%), and peripheral sensory neuropathy (grade 1 to $2: 56 \%$ ). Four patients had partial responses, including 2 patients with taxane-resistant breast cancer. ${ }^{13}$ These results were replicated in several additional studies with similar dosing schedules - ixabepilone day 1 every 21 days as an IV infusion. The MTD/recommended phase II dose was $40 \mathrm{mg} / \mathrm{m}^{2}$ in 2 studies $^{14,15}$ and $50 \mathrm{mg} / \mathrm{m}^{2}$ in a third study. ${ }^{16}$ Significant toxicities ( $\geq$ grade 3 ) included neutropenia ( $22 \%$ to $42 \%$ ), peripheral sensory neuropathy (13\%), fatigue (13\% to $22 \%)$, and gastrointestinal distress (5\% to $7 \%) .{ }^{14-16}$

Daily dosing for 3 to 5 consecutive days of a 21-day cycle has also been evaluated. A study of ixabepilone as an IV infusion for 3 consecutive days every 21 days included a total of 26 patients, 4 of whom had breast cancer. The MTD was determined to be $8 \mathrm{mg} / \mathrm{m}^{2} /$ day with a recommended phase II dose of 8 to $10 \mathrm{mg} / \mathrm{m}^{2} /$ day. The primary DLT was neutropenia $\geq$ grade $3 .{ }^{17}$ Another study evaluated MTD for ixabepilone as an IV infusion for 5 consecutive days every 21 days. Twenty-seven patients were enrolled on study, including 4 patients with breast cancer. The MTD was $6 \mathrm{mg} / \mathrm{m}^{2} /$ day; the primary DLT was neutropenia $\geq$ grade $3 .{ }^{18}$

In addition to single-agent phase I studies, ixabepilone has also been evaluated in combination with fixed-dose gemcitabine for patients with advanced solid tumors. Gemcitabine 


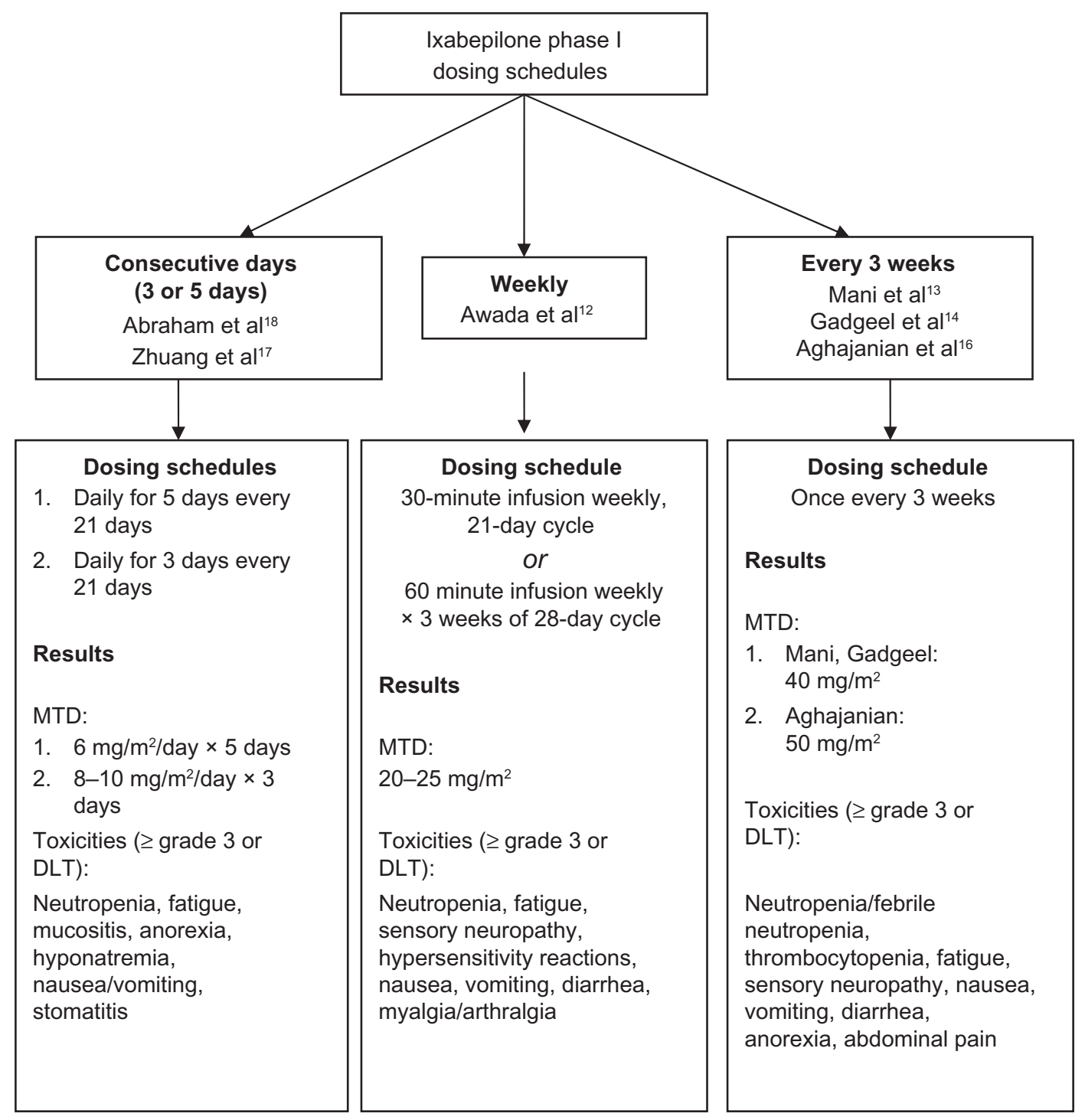

Figure I Phase I trials of single-agent ixabepilone.

Abbreviation: DLT, dose-limiting toxicities; MTD, maximum tolerated dose/recommended phase II dose.

in combination with paclitaxel demonstrated efficacy in metastatic breast cancer; given this information, the combination with ixabepilone was logical. ${ }^{19,20}$ The majority of patients (65\%) had prior taxane therapy; none of the patients, however, had breast cancer. Each treatment cycle was 21 days: gemcitabine 750 or $900 \mathrm{mg} / \mathrm{m}^{2}$ days 1 and 8 with ixabepilone IV infusion over 3 hours on day 8 . The MTD was gemcitabine $900 \mathrm{mg} / \mathrm{m}^{2}$ days 1 and 8 with ixabepilone $20 \mathrm{mg} / \mathrm{m}^{2}$ day 8 . The primary toxicity reported was myelosuppression with grade 3 or higher neutropenia occurring in the majority of patients (95\%); neutropenic fever, however, was uncommon (1 patient). Additional grade 3 toxicities included fatigue, hyponatremia, hypokalemia, thrombosis, and hypersensitivity reaction. Although myelosuppression was observed in other early phase studies of ixabepilone, the higher incidence noted in this study was likely related to the combination with gemcitabine. Moreover, it was noted that the lower gemcitabine dose $\left(740 \mathrm{mg} / \mathrm{m}^{2}\right)$ was still associated with higher rates of myelosuppression. In the evaluable patients, stable disease was the best response observed ( 9 of 14 patients, $64 \%) .{ }^{21}$

The multitude of phase I studies have also provided important pharmacologic data on ixabepilone. Pharmacokinetics for all dosing schedules dosing demonstrated a rapid dose decline in the first hour after dose completion, followed by a prolonged elimination phase, ${ }^{18}$ mean half-lives ranged from 16.8 hours for daily dosing ${ }^{18}$ to 38 to 40 hours for dosing once every 3 weeks. ${ }^{13,22}$ Ixabepilone is metabolized extensively in the liver with involvement by cytochrome P450. Ixabepilone metabolism is impaired by CYP3A4 
inhibitors; medications that affect these cytochrome systems are not recommended for use with this therapy. ${ }^{23}$ Ixabepilone is eliminated primarily as inactive metabolites in the feces and urine. Due to its liver metabolism, dose adjustments are required for mild hepatic impairment, and ixabepilone is not recommended for patients with significant hepatic dysfunction. ${ }^{7,13,16,18,24}$

\section{Efficacy as monotherapy in taxane-resistant breast cancer}

Ixabepilone as a single agent has been studied in patients with disease resistant to multiple agents and as an early therapy in metastatic disease. The current United States Food and Drug Administration (FDA) approval is for taxane- and anthracycline-resistant metastatic or locally advanced disease only (Table 1).

\section{Resistant disease}

A phase II, single-arm, multicenter trial lead to the approval by the FDA of ixabepilone as monotherapy for multi-drug resistant metastatic breast cancer. Eligible patients were women over 18 years of age with progressive metastatic disease despite therapy with anthracyclines, taxanes, and capecitabine. The eligible patients were also considered resistant to prior treatments, defined as progression within 8 weeks after therapy in the metastatic setting or recurrence within 6 months in the adjuvant or neoadjuvant setting. Patients with estrogen and progesterone receptor (ER/PR) positive disease were required to have failed hormone therapy; likewise, human epidermal growth receptor 2 (Her-2)-positive patients must have progressed despite trastuzumab. Patients with neither ER/PR nor Her-2 receptors, so-called "triple-negative" cancers, were also enrolled. ${ }^{25}$ This subpopulation of breast cancer patients accounts for approximately $15 \%$ of breast cancers and generally tends to have a worse prognosis. ${ }^{26}$ Prior treatment was limited to 5 chemotherapy regimens, with only 3 prior chemotherapy regimens in the metastatic setting. Significant exclusions were patients with brain metastases, pre-existing neuropathy (grade 2 or higher), and prior treatment with epothilones.

The study enrolled 126 patients (113 patients were assessable for response) from multiple centers in 10 different countries, including the United States. The median patient age was 51 years old and most had excellent performance status (96\% of patients with Karnofsky performance score $\geq 80$ ). Triple-negative patients (ER/PR/Her-2 negative) patients comprised $33 \%$ of the study population; only $7 \%$ of patients were Her-2 positive. Most patients had lung or liver metastases $(77 \%)$.

Treatment consisted of ixabepilone $40 \mathrm{mg} / \mathrm{m}^{2}$ monotherapy administered on day 1 of a 21 -day cycle. The patients received a median of 4 cycles of therapy; one-quarter of the patients $(25 \%)$ received at least 8 cycles.

The therapy was generally well-tolerated. Myelosuppression, particularly leukopenia and anemia, and peripheral neuropathy were the most common observed toxicities. Grade 3 or 4 neutropenia was observed in 68 patients (54\%); febrile neutropenia and infection, however, were only reported in seven patients. Peripheral sensory neuropathy grade 2 or higher developed in $60 \%$ of patients. Of these patients, 17 patients (13\%) developed grade 3 or 4 neuropathy. Resolution of peripheral neuropathy $\geq$ grade 3 occurred in 13 of the 17 patients after cessation of chemotherapy.

Objective response rate was the primary objective of the study. Both the investigator and an independent radiology facility reviewed all responses. Partial response rate was $11.5 \%$ (95\% confidence interval [CI], 6.3\% to $18.9 \%$ ) by the independent reviewer. Stable disease was achieved in $50 \%$ of the patients; $13.3 \%$ (95\% CI, $7.6 \%$ to $20.9 \%$ ) of assessable patients had stable disease for 6 months or more. Progression-free survival was 3.1 months with a median overall survival of 8.6 months. ${ }^{25}$ Similar response rates in anthracycline-resistant metastatic breast cancer have been reported with capecitabine $(36 \%),{ }^{27}$ paclitaxel $(22 \%$ to $28 \%),{ }^{27,28}$ docetaxel $(29 \%-41 \%),{ }^{29,30}$ and vinorelbine $(15 \%$ to $16 \%)^{31,32}$

Similar survival and response rates were reported in another phase II study which evaluated ixabepilone as a single agent in taxane-resistant breast cancer. This international study enrolled 66 patients; only 49 patients received the standard dose of $40 \mathrm{mg} / \mathrm{m}^{2}$ and were evaluated for toxicity. All of the patients had received prior surgical therapy, and the majority had received radiation and hormonal therapy. Taxane therapy (docetaxel or paclitaxel) was included in the most recent metastatic treatment regimen in $98 \%$ of patients; $73 \%$ of patients had progressed within 1 month of the most recent chemotherapy. The initial dosing schedule was $50 \mathrm{mg} / \mathrm{m}^{2}$ every 21 days. Subsequent adjustments were made based on toxicity and phase I data; the majority of patients received $40 \mathrm{mg} / \mathrm{m}^{2}$ every 21 days. Patients received a median of 3 cycles of therapy. In the intention to treat analysis, including all 66 patients, objective tumor response rate was $12 \%$ (95\% CI, $5.4 \%$ to $22.5 \%$ ) with all responders achieving partial response. Twenty-eight patients $(42 \%)$ achieved stable 

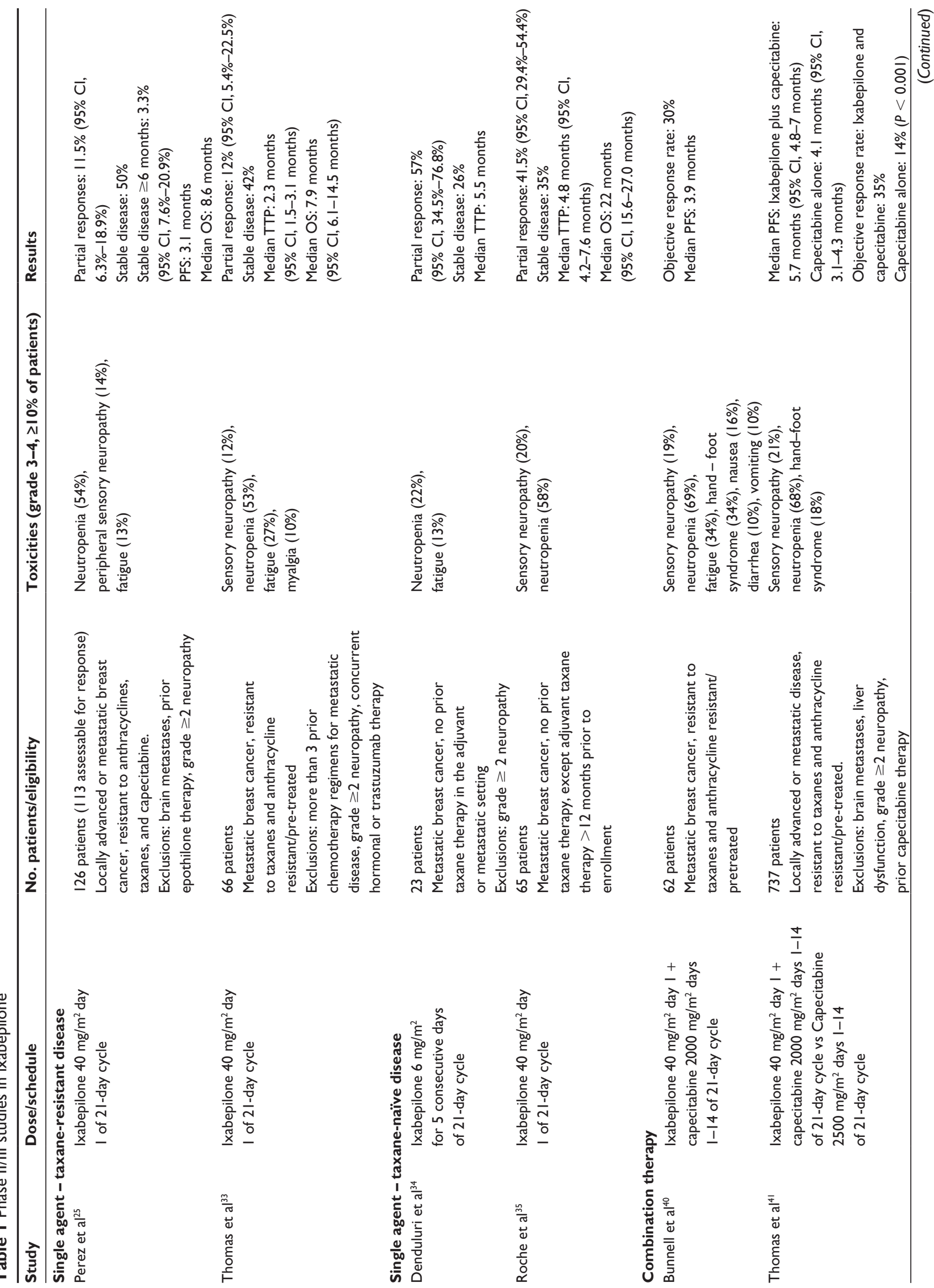


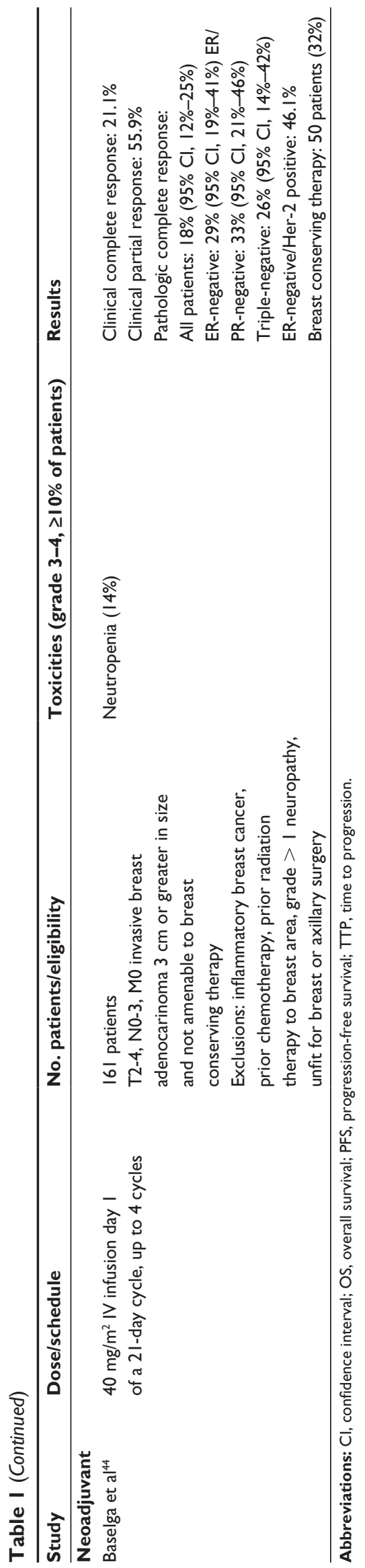

disease as best response. Among the 49 patients who received ixabepilone $40 \mathrm{mg} / \mathrm{m}^{2}, 20$ patients had stable disease (41\%). The majority of the patients with stable disease $(80 \%)$ received at least 4 cycles of ixabepilone therapy. Median time to progression was 2.3 months ( $95 \%$ CI, 1.5 to 3.1 months). Median survival for the patients receiving ixabepilone $40 \mathrm{mg} / \mathrm{m}^{2}$ was 7.9 months ( $95 \%$ CI, 6.1 to 14.5 months). Frequent and serious toxicities ( $\geq$ grade 3 ) included neutropenia (53\%), febrile neutropenia ( $6 \%)$, sensory neuropathy $(12 \%)$, myalgias $(10 \%)$, and fatigue $(27 \%){ }^{33}$

As a single agent in a patient population with disease refractory to standard chemotherapy, ixabepilone demonstrated efficacy with tolerable toxicity. The approval of ixabepilone in this setting provides an option to prolong both progression-free and overall survival in patients with resistant disease.

\section{Efficacy as monotherapy in taxane-naïve breast cancer}

Given the efficacy demonstrated with ixabepilone in patients with taxane-resistant or refractory breast cancer, use as monotherapy in place of taxane therapy has been investigated in several studies.

The United States National Cancer Institute conducted a phase II study investigating the use of ixabepilone in taxanenaïve patients. Ixabepilone was administered at a dose of $6 \mathrm{mg} / \mathrm{m}^{2}$ for 5 consecutive days every 21 days. Twenty-three patients were enrolled; eligibility was limited to metastatic breast cancer patients who did not receive taxane therapy in the adjuvant or metastatic setting. No other limitations were placed on prior chemotherapy: 12 patients $(52 \%)$ had received prior anthracycline therapy, 13 patients $(57 \%)$ had received prior hormonal therapy, and 5 patients $(22 \%)$ of patients had no prior therapy. The median number of prior therapies was not reported. Patients received a median of 8 cycles (range 2 to 22 cycles) of therapy. Four patients required dose reductions due to toxicity.

The primary objective of the study was to assess efficacy as measured by response rate. The objective response rate was $57 \%$ (95\% CI, 34.5\% to $76.8 \%$ ) with 13 partial responses; the median duration of response was 5.6 months. No complete responses were observed. Stable disease for at least 6 weeks was the best response in 6 patients $(26 \%)$. The median time to progression for all patients was 5.5 months.

Toxicities included myelosuppression, fatigue, diarrhea, and sensory neuropathy. Grade 1 to 2 neutropenia and anemia occurred in over $60 \%$ of patients; grade 3 or 4 neutropenia occurred in $22 \%$ of patients. No febrile neutropenia was 
observed, but one patient required filgrastim due to prolonged neutropenia. Neuropathy was common and primarily sensory: grade 1 or 2 sensory neuropathy occurred in $42 \%$ of patients. In addition, 2 patients developed grade 2 to 3 motor neuropathy. ${ }^{34}$

An international trial also investigated the use of ixabepilone as first-line metastatic chemotherapy in patients with prior adjuvant anthracycline exposure. Prior adjuvant taxane therapy was allowed if at least 12 months had elapsed since the last dose, but only 11 patients $(17 \%)$ had received prior taxane therapy. Sixty-five patients were enrolled. The vast majority had received 1 prior anthracycline regimen (60 patients, 92\%), and 5 patients had received 2 prior anthracycline regimens. The dosing schedule of ixabepilone was $40 \mathrm{mg} / \mathrm{m}^{2}$ IV infusion over 3 hours on day 1 of a 21 -day cycle; the patients received a median of 6 treatment cycles. Eighteen patients $(28 \%)$ required dose reduction from the starting dose of $40 \mathrm{mg} / \mathrm{m}^{2}$.

The primary endpoint of the study was objective response rate, while median survival, time to progression, and response duration were secondary endpoints. The overall response rate was $41.5 \%$ (95\% CI, $29.4 \%$ to $54.4 \%$ ) with all partial responses. The median duration of response was 8.2 months (95\% CI, 5.7 to 10.2 months). Twenty-three patients (35\%) had stable disease as their best response. The median time to progression was 4.8 months ( $95 \%$ CI, 4.2 to 7.6 months) with a median survival of 22 months (95\% CI, 15.6 to 27.0 months).

As with prior studies, neuropathy and myelosuppression were the most common serious toxicities. Most patients had grade 1 or 2 sensory neuropathy (51\%), but grade 3 sensory neuropathy was observed in 13 patients $(20 \%)$. Motor neuropathy (grade 2 to 3 ) was also observed in 4 patients. Myelosuppression was a frequent toxicity, occurring in more than $90 \%$ of patients; neutropenia $\geq$ grade 3 was observed in $58 \%$ of patients. Additional toxicities $\geq$ grade 3 included fatigue, nausea, vomiting, and myalgias. ${ }^{35}$

The response rates in this setting are comparable to patients receiving paclitaxel or docetaxel and are encouraging for further investigation. A recent Eastern Oncology Cooperative Group phase III study, E2100, evaluated paclitaxel with or without bevacizumab, recombinant human vascular endothelial growth factor monoclonal antibody. Results demonstrated statistically significant improvement in progression free survival and overall response rates with combination therapy. ${ }^{36,37}$ Given these encouraging results, an ongoing phase III Cancer and Leukemia Group B (CALGB) study is evaluating the combination of paclitaxel, ixabepilone, or nanoparticle albumin bound paclitaxel plus bevacizumab as first-line therapy for metastatic disease. The rationale for comparing these 3 regimens is to understand the relative benefits and toxicities of weekly ixabepilone and nab-paclitaxel when compared to weekly paclitaxel. Nab-paclitaxel is theorized to have improved tumor penetration via nanoparticles and therefore improved efficacy. ${ }^{38,39}$ Ixabepilone, as discussed in this paper, has demonstrated efficacy in taxane-resistant and taxanenaïve patients. Comparing the efficacy of these 2 agents with the standard weekly paclitaxel and bevacizumab will help to identify if there is one agent that results in better efficacy and improved toxicity when combined with bevacizumab as first-line therapy in metastatic breast cancer. Progression-free survival is the primary endpoint and other pharmacodynamic and pharmacogenomic endpoints will also be evaluated. Toxicities will be also evaluated as a secondary endpoint; in particular, rates of grade 3 to 4 sensory neuropathy and peripheral neuropathy will be assessed by Functional Assessment of Cancer Therapy/Gynecologic Oncology Group-Neurotoxicity subscale to determine the relative impact of these combinations on clinically relevant neurotoxicity. The results of this trial will answer important questions concerning the use of these microtubule targeted agents with bevacizumab in the first line setting.

\section{Combination therapy}

Ixabepilone has also been studied in combination with capecitabine. A phase I/II study identified the MTD for the combination of ixabepilone and capecitabine in patients with taxane-resistant breast cancer. For the phase I portion of the study, 74 patients were treated on 2 different dosing schedules. On schedule A, ixabepilone $40 \mathrm{mg} / \mathrm{m}^{2}$ IV infusion was given day 1 with escalating dose of capecitabine from $1650 \mathrm{mg} / \mathrm{m}^{2}$ to $2000 \mathrm{mg} / \mathrm{m}^{2}$ orally daily on days 1 to 14 of a 21-day cycle. Schedule B consisted of ixabepilone $8 \mathrm{mg} / \mathrm{m}^{2}$ to $10 \mathrm{mg} / \mathrm{m}^{2} \mathrm{IV}$ infusion days 1 to 3 with capecitabine $1650 \mathrm{mg} / \mathrm{m}^{2}$ orally daily days 1 to 14 of a 21 -day cycle. No dose-limiting toxicities were observed on dosing schedule B. The maximum tolerated dose for schedule A was defined as ixabepilone $40 \mathrm{mg} / \mathrm{m}^{2}$ with capecitabine $2000 \mathrm{mg} / \mathrm{m}^{2}$ daily and this dosing schedule was tested for efficacy. In the phase II portion of the study, 62 patients were treated. The objective response rate was $30 \%$; median progression-free survival was 3.9 months. Peripheral sensory neuropathy, myelosuppression, fatigue were among the dose-limiting toxicities. These results demonstrated acceptable safety and efficacy for this combination in taxane-resistant metastatic breast cancer. ${ }^{40}$

Ixabepilone and capecitabine in combination were then studied in an international, open-label, phase III trial. Eligible 
patients were women at least 18 years of age with locally advanced or metastatic breast cancer. These patients were taxane resistant and either resistant to or pre-treated with anthracycline therapy. Resistance was defined as tumor progression during therapy or within 3 to 4 months for metastatic disease and 6 to 12 months in the adjuvant setting. Patients who had received a minimum cumulative anthracycline dose (doxorubicin $240 \mathrm{mg} / \mathrm{m}^{2}$ or epirubicin $360 \mathrm{mg} / \mathrm{m}^{2}$ ) were also eligible. Pertinent exclusion criteria included brain metastases, liver dysfunction, neuropathy grade 2 or higher, and prior capecitabine therapy.

The international study randomized 737 patients to either ixabepilone and capecitabine or capecitabine alone. The majority of patients had multiple metastatic sites, including liver and/or lung involvement. Triple negative (ER/PR/Her-2 negative) patients accounted for $24 \%$ of the combination therapy group and $26 \%$ of the single-agent therapy group; Her-2-positive patients accounted for $15 \%$ of the total study population. Pre-treatment was extensive: $41 \%$ of patients in the combination arm and $37 \%$ in the capecitabine alone arm had received 2 prior chemotherapy regimens in the metastatic setting. Ninety-seven percent of patients in both groups had received prior anthracyclines. Likewise, $96 \%$ to $98 \%$ of patients in each group had received taxane therapy and had either progressed or become resistant to therapy.

Treatment was administered in 21-day cycles and continued until disease progression or unacceptable toxicity. The dosing schedule for combination therapy was ixabepilone $40 \mathrm{mg} / \mathrm{m}^{2} \mathrm{IV}$ infusion over 3 hours on day 1 plus capecitabine $2000 \mathrm{mg} / \mathrm{m}^{2}$ orally days 1 to 14 of a 21-day cycle. Single-agent capecitabine was administered at $2500 \mathrm{mg} / \mathrm{m}^{2}$ orally days 1 to 14 of a 21-day cycle. Crossover from capecitabine alone to combination therapy was not allowed. For ixabepilone plus capecitabine, a median of 5 cycles were given; for capecitabine alone, a median of 4 cycles were given. Dose reductions were relatively uncommon. In the combination therapy arm, $88 \%$ and $62 \%$ received $\geq 70 \%$ of their relative ixabepilone and capecitabine dose intensity, respectively. In the singleagent arm, $82 \%$ of patients received $\geq 70 \%$ of their relative capecitabine dose intensity $\left(2500 \mathrm{mg} / \mathrm{m}^{2}\right) .{ }^{41}$ The differential dosing of capecitabine in both arms was also used in another trial in metastatic breast cancer where capecitabine alone was a comparator. ${ }^{42}$ The rationale for using lower dose of capecitabine given in the combination arm is to reduce toxicity and improve dose-intensity. Interestingly as described below, despite the lower dose of capecitabine in the combination arm, the efficacy was improved. ${ }^{41}$
Significant toxicities (grade 3 or 4) included neuropathy, hand - foot syndrome, myelosuppresion, fatigue, diarrhea, and myalgias. Peripheral sensory neuropathy occurred in $65 \%$ of patients with ixabepilone therapy; $21 \%$ of these patients developed neuropathy grade 3 or higher. Incidence of grade 3 hand - foot syndrome was similar in both treatment arms (18 vs 17\%). Myelosuppression was more common in the combination therapy group; grade 3 to 4 neutropenia was observed in $68 \%$ of patients with combination therapy and $11 \%$ with capecitabine alone. Growth factor support was used in $20 \%$ of patients receiving combination therapy and $3 \%$ of patients on capecitabine alone. The incidence of fatigue, diarrhea, and myalgias were similar in both groups.

Progression-free survival was the primary endpoint of the study; secondary endpoints included tumor response rate, duration of overall response, patient symptom assessment, and overall survival. Median progression-free survival was improved to 5.7 months (95\% CI, 4.8 to 6.7 months) for ixabepilone plus capecitabine versus 4.1 months (95\% CI, 3.1 to 4.3 months) for capecitabine alone. The hazard ratio indicated a $31 \%$ reduction in risk of disease progression, favoring combination therapy. Objective response rate was also improved with combination therapy: $35 \%$ for ixabepilone and capecitabine versus $14 \%$ for capecitabine alone $(P<0.001)$. Overall survival data are not yet available.

Pre-planned subgroup analysis of progression-free survival was also performed. The subgroups were designed to evaluate the impact of potential prognostic factors: age, race, performance status, visceral disease involvement, prior therapy, hormone receptor status, and Her-2 receptor status. Benefit, as measured by longer progression-free survival, was maintained across the subgroups. Patients with liver dysfunction, defined as liver function tests $\geq$ grade 2 , were the only exception. An increased rate of death for patients receiving combination therapy was noted in this small group of patients $(31 \%$ combination therapy versus $19 \%$ singleagent capecitabine, hazard ratio 2.61)..$^{41,43}$

Based on these clinical trials, the combination of ixabepilone $40 \mathrm{mg} / \mathrm{m}^{2}$ day 1 with capecitabine $2000 \mathrm{mg} / \mathrm{m}^{2}$ daily days 1 to 14 of a 21-day cycle is approved for patients with locally advanced or metastatic breast cancer who have failed taxane and anthracycline therapy.

\section{Neoadjuvant chemotherapy}

A phase II study of ixabepilone in the neoadjuvant setting has explored efficacy, safety, and predictors of response. The primary objective of the trial was a preclinical analysis of potential predictors of response to ixabepilone. Secondary 
endpoints included rate of complete pathologic response (pCR), clinical and radiologic responses, safety, and the proportion of patients able to have breast conserving therapy. The preclinical work discussed in this trial is beyond the scope of this review and will not be discussed. ${ }^{44}$

Eligible patients included women with T2-4, N0-3, M0 invasive breast adenocarinoma $3 \mathrm{~cm}$ or greater in size and not amenable to breast conserving therapy. Patients with inflammatory breast cancer, baseline neuropathy of $\geq$ grade 1 , or non-surgical candidates were excluded. One hundred sixty-one patients were enrolled. Triple-negative (ER/PR/ Her-2 negative) patients accounted for $26 \%$ of the study population.

The treatment plan was for ixabepilone $40 \mathrm{mg} / \mathrm{m}^{2} \mathrm{IV}$ infusion over 3 hours on day 1 of a 21-day cycle, up to 4 cycles. Most of the patients ( $88 \%$ ) received 4 cycles of therapy; only $7 \%$ of the patients required dose reductions.

Clinical complete response was observed in $21.1 \%$ and partial response in $55.9 \%$ of patients. The independently assessed pCR in the breast was 14\% (95\% CI, 9\% to 20\%). Higher rates of pCR were observed in hormone-negative and Her-2-negative patients: $29 \%$ (95\% CI, 19\% to $41 \%$ ) in ER-negative, 33\% (95\% CI, $21 \%$ to $46 \%$ ) in ER/PR-negative, and $26 \%(95 \% \mathrm{CI}, 14 \%$ to $42 \%)$ in triple-negative (ER/ PR/Her-2 negative). Although there were only 13 patients, ER-negative/HER-2-positive patients had the best pathologic response rate $(46.1 \%)$. The study does not report whether Her-2-positive patients also received trastuzumab therapy in the neoadjuvant setting. ${ }^{44}$ Surgery was performed after four cycles of therapy in 154 of the 161 patients; breast conserving therapy, however, was performed in only 50 patients $(32 \%){ }^{44}$

\section{Toxicity and tolerability}

Ixabepilone belongs to the family of microtubule-stabilizing chemotherapeutic agents and is closely related to the taxanes paclitaxel and docetaxel. As a result, the toxicity profile for ixabepilone is similar to those of these agents.

Myelosuppression and peripheral neuropathy are the most common toxicities reported in phase II/III clinical trials using ixabepilone. Neutropenia is relatively common with a reported incidence in $79 \%$ to $89 \%$ of patients (all grades) and $53 \%$ to $58 \%$ with grade 3 or 4 . Anemia is reported in up to $92 \%$ of patients (all grades) with only $3 \%$ to $8 \%$ grade 3 or 4 . Despite the high incidence of neutropenia, febrile neutropenia and infection grade 3 or 4 is reported in $\leq 10 \%$ of patients receiving ixabepilone monotherapy. ${ }^{7,25,33,35}$ Similar rates of myelosuppression are reported with the combination of ixabepilone and capecitabine: neutropenia in $89 \%$ of patients with $68 \%$ grade 3 or 4 and febrile neutropenia in $5 \%$ of patients. ${ }^{41}$

Peripheral neuropathy is also common and appears to be cumulative, consistent with observations in taxane therapy. The neuropathy observed is primarily sensory, although motor neuropathy has been reported. Peripheral sensory neuropathy of all grades associated with ixabepilone therapy (monotherapy or in combination) was observed in up to $70 \%$ of patients with grade 3 or 4 neuropathy in up to $20 \%$ of patients. ${ }^{25,33,35,41}$ Sensory neuropathy is generally considered reversible when the drug is discontinued, although this is not always the case. No definitive preventive therapy has been developed. As with taxane therapy, older age, pre-existing neuropathy, and co-morbid conditions such as diabetes mellitus appear to increase the risk of significant neuropathy. Overall, rates of sensory neuropathy with ixabepilone are comparable to those seen with paclitaxel. ${ }^{45}$

Additional common toxicities include fatigue, myalgias, arthralgias, alopecia, gastrointestinal disturbances (nausea, vomiting, diarrhea, constipation, abdominal pain), mucositis, and nail changes. ${ }^{25,33,35,41}$ These toxicities are generally manageable and not dose-limiting. Due to the Cremophor $\mathrm{EL}^{\circledR}$ (polyoxyethylated castor oil) component of the IV infusion, hypersensitivity reactions, including anaphylaxis, were reported in $1 \%$ of patients on clinical trials. To prevent this complication, pre-medication is recommended with $\mathrm{H} 1$ and $\mathrm{H} 2$ antagonists (diphenhydramine and ranitidine). ${ }^{24}$

In general, ixabepilone has a similar toxicity profile to taxanes (docetaxel, paclitaxel). Myelosuppression, although common, is infrequently associated with complications such as febrile neutropenia. At this time, routine growth factor support is not recommended. ${ }^{7}$ Peripheral neuropathy requires close monitoring and dose adjustments if and when symptoms develop. ${ }^{7,45}$

\section{Future directions}

Ixabepilone remains a drug of interest for the treatment of breast cancer. Multiple clinical trials are ongoing to further define the role of this medication. Areas of interest include use of ixabepilone for triple-negative breast cancer, substitution of ixabepilone for taxanes in the adjuvant and neoadjuvant settings, and combining ixabepilone with newer agents (the tyrosine kinase inhibitors bevacizumab, trastuzumab). In addition, development of novel epothilone compounds is ongoing and may broaden clinical use both in breast cancer specifically and solid tumors in general.

Clinical trials in several different areas are ongoing. Neoadjuvant therapy trials include the combination of 
carboplatin, ixabepilone, and trastuzumab for Her-2 positive patients and ixabepilone with cyclophosphamide for Her-2-negative patients. In the adjuvant setting, ixabepilone is being compared to both paclitaxel and docetaxel. As first line therapy for metastatic disease, an ongoing CALGB trial is comparing paclitaxel, nanoparticle albumin bound paclitaxel, and ixabepilone in combination with bevacizumab for patients with locally advanced or metastatic breast cancer as described earlier in the article. ${ }^{46}$ For second-line therapy and beyond in breast cancer, combinations of ixabepilone with dasatinib, ${ }^{47}$ sorafenib, ${ }^{48}$ and hydroxychloroquine ${ }^{49}$ are being tested.

\section{Conclusion}

The addition of novel chemotherapeutic agents and targeted therapies has improved outcomes in breast cancer over the last decade. Anthracyclines and taxanes have become the cornerstone of systemic management of breast cancer. Unfortunately development of resistance to these agents results in failure of therapy. Ixabepilone is a promising new microtubule stabilizing agent that has shown efficacy in advanced resistant breast cancer due to its low susceptibility to multiple drug resistant mechanisms. Moreover, it has shown synergistic activity when combined with capecitabine. Hence this agent has become an exciting new addition to the armamentarium of agents used in breast cancer.

Several unanswered questions still exist: the efficacy of this drug earlier in the management of breast cancer; whether it will prevent or delay the development of resistance; whether combination of ixabepilone with targeted therapies such as trastuzumab and bevacizumab will result in improved outcomes; whether there is a dosing schedule of ixabepilone that will reduce the incidence of the common toxicities of neuropathy and myelosuppression; the mechanism of development of resistance to ixabepilone therapy; and whether there are reliable molecular biomarkers that can predict resistance to ixabepilone therapy. Clinical trials are ongoing to answer some of these questions.

\section{Disclosures}

The authors declare no conflicts of interest.

\section{References}

1. Jemal A, Siegel R, Ward E, Hao Y, Xu J, Thun MJ. Cancer Statistics, 2009. CA Cancer J Clin. 2009;59(4):225-249.

2. Hamilton A, Hortobagyi G. Chemotherapy: what progress in the last 5 years? J Clin Oncol. 2005;23(8):1760-1775.

3. Bollag DM, McQueney PA, Zhu J, et al. Epothilones, a new class of microtubule-stabilizing agents with a taxol-like mechanism of action. Cancer Res. 1995;55(11):2325-2333.
4. Gerth K, Bedorf N, Hofle G, Irschik H, Reichenbach H. Epothilons A and B: antifungal and cytotoxic compounds from Sorangium cellulosum (Myxobacteria). Production, physico-chemical and biological properties. J Antibiot (Tokyo). 1996;49(6):560-563.

5. Lee FY, Borzilleri R, Fairchild CR, et al. BMS-247550: a novel epothilone analog with a mode of action similar to paclitaxel but possessing superior antitumor efficacy. Clin Cancer Res. 2001;7(5): 1429-1437.

6. Lee FY, Borzilleri R, Fairchild CR, et al. Preclinical discovery of ixabepilone, a highly active antineoplastic agent. Cancer Chemother Pharmacol. 2008;63(1):157-166.

7. Boehnke Michaud L. The optimal therapeutic use of ixabepilone in patients with locally advanced or metastatic breast cancer. $J$ Oncol Pharm Pract. 2009; 15(2):95-106.

8. Nettles JH, Li H, Cornett B, Krahn JM, Snyder JP, Downing KH. The binding mode of epothilone A on alpha,beta-tubulin by electron crystallography. Science. 2004;305(5685):866-869.

9. Lee FY, Smykla R, Johnston K, et al. Preclinical efficacy spectrum and pharmacokinetics of ixabepilone. Cancer Chemother Pharmacol. 2009;63(2):201-212.

10. Dumontet C, Jordan MA, Lee FF. Ixabepilone: targeting betaIIItubulin expression in taxane-resistant malignancies. Mol Cancer Ther. 2009;8(1):17-25.

11. Rivera E, Lee J, Davies A. Clinical development of ixabepilone and other epothilones in patients with advanced solid tumors. Oncologist. 2008;13(12):1207-1223.

12. Awada A, Piccart MJ, Jones SF, et al. Phase I dose escalation study of weekly ixabepilone, an epothilone analog, in patients with advanced solid tumors who have failed standard therapy. Cancer Chemother Pharmacol. 2009;63(3):417-425.

13. Mani S, McDaid H, Hamilton A, et al. Phase I clinical and pharmacokinetic study of BMS-247550, a novel derivative of epothilone B, in solid tumors. Clin Cancer Res. 2004;10(4):1289-1298.

14. Gadgeel SM, Wozniak A, Boinpally RR, et al. Phase I clinical trial of BMS-247550, a derivative of epothilone B, using accelerated titration 2B design. Clin Cancer Res. 2005;11(17):6233-6239.

15. Shimizu T, Yamamoto N, Yamada Y, et al. Phase I clinical and pharmacokinetic study of 3-weekly, 3-h infusion of ixabepilone (BMS247550), an epothilone B analog, in Japanese patients with refractory solid tumors. Cancer Chemother Pharmacol. 2008;61(5):751-758.

16. Aghajanian C, Burris HA 3rd, Jones S, et al. Phase I study of the novel epothilone analog ixabepilone (BMS-247550) in patients with advanced solid tumors and lymphomas. J Clin Oncol. 2007;25(9):1082-1088.

17. Zhuang SH, Agrawal M, Edgerly M, et al. A phase I clinical trial of ixabepilone (BMS-247550), an epothilone B analog, administered intravenously on a daily schedule for 3 days. Cancer. 2005;103(9): 1932-1938.

18. Abraham J, Agrawal M, Bakke S, et al. Phase I trial and pharmacokinetic study of BMS-247550, an epothilone B analog, administered intravenously on a daily schedule for five days. J Clin Oncol. 2003;21(9): $1866-1873$.

19. Delfino C, Caccia G, Gonzales LR, et al. Gemcitabine plus paclitaxel as first-line chemotherapy for patients with advanced breast cancer. Oncology. 2004;66(1):18-23.

20. Demiray M, Kurt E, Evrensel T, et al. Phase II study of gemcitabine plus paclitaxel in metastatic breast cancer patients with prior anthracycline exposure. Cancer Invest. 2005;23(5):386-391.

21. Hensley ML, Dizon D, Derosa F, et al. A phase I trial of BMS- 247550 (NSC\# 710428) and gemcitabine in patients with advanced solid tumors. Invest New Drugs. 2007;25(4):335-341.

22. Plummer R, Woll P, Fyfe D, et al. A phase I and pharmacokinetic study of ixabepilone in combination with Carboplatin in patients with advanced solid malignancies. Clin Cancer Res. 2008;14(24):8288-8294.

23. Goel S, Cohen M, Comezoglu SN, et al. The effect of ketoconazole on the pharmacokinetics and pharmacodynamics of ixabepilone: a first in class epothilone B analogue in late-phase clinical development. Clin Cancer Res. 2008;14(9):2701-2709. 
24. Ixempra (ixabepilone) intravenous infusion package insert. In: Squibb BM, edPrinceton, NJ: Bristol-Myers Squibb.

25. Perez EA, Lerzo G, Pivot X, et al. Efficacy and safety of ixabepilone (BMS-247550) in a phase II study of patients with advanced breast cancer resistant to an anthracycline, a taxane, and capecitabine. J Clin Oncol. 2007;25(23):3407-3414.

26. Onitilo AA, Engel JM, Greenlee RT, Mukesh BN. Breast cancer subtypes based on ER/PR and Her2 expression: comparison of clinicopathologic features and survival. Clin Med Res. 2009;7(1-2):4-13.

27. Talbot DC, Moiseyenko V, Van Belle S, et al. Randomised, phase II trial comparing oral capecitabine (Xeloda) with paclitaxel in patients with metastatic/advanced breast cancer pretreated with anthracyclines. Br J Cancer. 2002;86(9):1367-1372.

28. Perez EA. Paclitaxel in breast cancer. Oncologist. 1998;3(6): 373-389.

29. Bonneterre J, Spielman M, Guastalla JP, et al. Efficacy and safety of docetaxel (Taxotere) in heavily pretreated advanced breast cancer patients: the French compassionate use programme experience. Eur J Cancer. 1999;35(10):1431-1439.

30. Trudeau ME. Phase I-II studies of docetaxel as a single agent in the treatment of metastatic breast cancer. Semin Oncol. 1999;263 Suppl 8: 21-26.

31. Degardin M, Bonneterre J, Hecquet B, et al. Vinorelbine (navelbine) as a salvage treatment for advanced breast cancer. Ann Oncol. 1994;5(5): 423-426.

32. Ibrahim NK, Rahman Z, Valero V, et al. Phase II study of vinorelbine administered by 96-hour infusion in patients with advanced breast carcinoma. Cancer.1999;86(7):1251-1257.

33. Thomas E, Tabernero J, Fornier M, et al. Phase II clinical trial of ixabepilone (BMS-247550), an epothilone B analog, in patients with taxane-resistant metastatic breast cancer. J Clin Oncol. 2007;25(23): 3399-3406.

34. Denduluri N, Low JA, Lee JJ, et al. Phase II trial of ixabepilone, an epothilone $\mathrm{B}$ analog, in patients with metastatic breast cancer previously untreated with taxanes. J Clin Oncol. 2007;25(23):3421-3427.

35. Roche H, Yelle L, Cognetti F, et al. Phase II clinical trial of ixabepilone (BMS-247550), an epothilone B analog, as first-line therapy in patients with metastatic breast cancer previously treated with anthracycline chemotherapy. J Clin Oncol. 2007;25(23):3415-3420.

36. Miller KD. E2100: a phase III trial of paclitaxel versus paclitaxel/ bevacizumab for metastatic breast cancer. Clin Breast Cancer. 2003;3(6): 421-422.

37. Gray R, Bhattacharya S, Bowden C, Miller K, Comis RL. Independent review of E2100: a phase III trial of bevacizumab plus paclitaxel versus paclitaxel in women with metastatic breast cancer. J Clin Oncol. 2009;27(30):4966-4972.
38. Carver LA, Schnitzer JE. Caveolae: mining little caves for new cancer targets. Nat Rev Cancer. 2003;3(8):571-581.

39. Desai N, Trieu V, Yao Z, et al. Increased antitumor activity, intratumor paclitaxel concentrations, and endothelial cell transport of cremophor-free, albumin-bound paclitaxel, ABI-007, compared with cremophor-based paclitaxel. Clin Cancer Res. 2006;12(4): 1317-1324.

40. Bunnell C, Vahdat L, Schwartzberg L, et al. Phase I/II study of ixabepilone plus capecitabine in anthracycline-pretreated/resistant and taxane-resistant metastatic breast cancer. Clin Breast Cancer. 2008;8(3):234-241.

41. Thomas ES, Gomez HL, Li RK, et al. Ixabepilone plus capecitabine for metastatic breast cancer progressing after anthracycline and taxane treatment. J Clin Oncol. 2007;25(33):5210-5217.

42. Geyer CE, Forster J, Lindquist D, et al. Lapatinib plus capecitabine for HER2-positive advanced breast cancer. $N$ Engl J Med. 2006;355(26):2733-2743.

43. Thomas ES. Ixabepilone plus capecitabine for metastatic breast cancer progressing after anthracycline and taxane treatment. J Clin Oncol. 2008;26(13):2223.

44. Baselga J, Zambetti M, Llombart-Cussac A, et al. Phase II genomics study of ixabepilone as neoadjuvant treatment for breast cancer. J Clin Oncol. 2009;27(4):526-534.

45. Lee JJ, Swain SM. Peripheral neuropathy induced by microtubulestabilizing agents. J Clin Oncol. 2006;24(10):1633-1642.

46. Rugo H, Lyss A, Moreno Aspitia A. Phase III randomized study of paclitaxel versus paclitaxel albumin-stabilized nanoparticle formulation versus ixabepilone in combination with bevacizumab in patients with locally recurrent, stage IIIB or IV breast cancer 2008; http:// www.cancer.gov/search/ViewClinicalTrials.aspx?cdrid $=617539 \&$ version $=$ HealthProfessional\&protocolsearchid=7150845\#Objectives _ CDR0000617539. Accessed October 20, 2009.

47. Schwartzberg LS. Trial of dasatinib plus ixabepilone in 2 nd or 3 rd line metastatic breast cancer http://www.cancer.gov/search/ ViewClinicalTrials.aspx?cdrid $=647959 \&$ version $=$ HealthProfessional $\&$ protocolsearchid=7150845. Accessed October 20, 2009.

48. Yardley DA. Phase I/II Trial of sorafenib plus ixabepilone in Her2negative metastatic breast cancer. 2009; http://www.cancer.gov/search/ ViewClinicalTrials.aspx?cdrid=633738\&version=HealthProfessional $\&$ protocolsearchid=7150845. Accessed October 20, 2009.

49. Karantza-Wadsworth V. Phase I/II study of ixabepilone and hydroxychloroquine in patients with taxane- and anthracycline-resistant metastatic breast cancer 2008; http://www.cancer.gov/search/ ViewClinicalTrials.aspx?cdrid=615000\&version=HealthProfessional $\&$ protocolsearchid=7150845\#ContactInfo_CDR0000615000. Accessed October 20, 2009.
Breast Cancer: Targets and Therapy

\section{Publish your work in this journal}

Breast Cancer: Targets and Therapy is an international, peerreviewed open access journal focusing on breast cancer research, identification of therapeutic targets and the optimal use of preventative and integrated treatment interventions to achieve improved outcomes, enhanced survival and quality of life for the cancer patient.

\section{Dovepress}

View the full aims and scopes of this journal here. The manuscript management system is completely online and includes a very quick and fair peer-review system, which is all easy to use. Visit http:// www.dovepress.com/testimonials.php to read real quotes from published authors. 